\title{
Acceso a la ficha clínica con fines de investigación biomédica
}

\author{
MANUEL OYARZÚN G.*
}

\section{Access to clinical records for biomedical research purposes}

The clinical information obtained from the clinical records is crucial for doing biomedical research. Up until some years ago a scarce limitation existed in the use of this information for research purposes. This situation has changed in nowadays because of the legal safeguarding of the privacy and confidentiality of personal data. In fact, article 13 of the Law 20.584 "Regulations of the rights and duties of health care users", states that people not related directly with patient care cannot have access to clinical records, with the exception of people with notarial authorization by the patient. Lately the following proposal has been done on this issue: in prospective studies, a consent from the patient and an authorization of the health authority should be required to access clinical records; in retrospective studies, consent should be obtained from the patient if his/her personal information is going to be used; if the information is nameless, the consent can be disregarded after being reviewed the protocol by a Research Ethics Committee. Ethical requirements of the collections of anonymized data and use of historical records of deceased subjects are also discussed. The role of the Research Ethics Committees as well as the Editorial Boards in observing and respecting the ethical regulations in the approval of research projects and in the acceptance of biomedical research publications has a major importance.

Key words: Clinical records; biomedical research; Research Ethical Committee; Editorial Board.

\section{Resumen}

La información que se puede obtener de la ficha clínica es de gran importancia para efectuar investigación biomédica. Hasta hace algunos años casi no existían limitaciones para usar esta información para fines de investigación. Actualmente esta situación ha cambiado debido a los resguardos legales que protegen la privacidad y confidencialidad de los datos personales. De hecho, el artículo 13 de la Ley 20.548 sobre derechos y deberes de los pacientes en su atención en salud, establece que las personas no relacionadas con la atención de la salud del paciente no pueden acceder a la ficha clínica, siendo una alternativa que el paciente otorgue una autorización notarial. Recientemente se ha hecho una proposición sobre este tema: para acceder a la fich a clínica en estudios prospectivos se requiere del consentimiento del paciente; en estudios retrospectivos, también se requiere consentimiento del paciente si se van a usar sus datos personales, en tanto que si la información es anonimizada, el consentimiento puede ser dispensado por el Comité de Ética de Investigación, luego de revisar el respectivo protocolo. Se discuten los requerimientos éticos de la recolección de datos anonimizados y el uso de registros históricos de sujetos fallecidos. El papel de los Comités de Ética de Investigación y de los Comités Editoriales es de la mayor importancia para mantener el debido respeto y observancia de las regulaciones éticas en la aprobación de proyectos de investigación y en las publicaciones de las investigaciones biomédicas.

Palabras clave: Ficha cínica; investigación biomédica; Comité de Ética de Investigación; Comité editorial.

* Profesor titular, Instituto de Ciencias Biomédicas y Presidente, Comité de Ética de Investigación en Seres Humanos. Facultad de Medicina, Universidad de Chile. 
El uso tradicional de la ficha clínica como un instrumento invaluable para acopiar información fundamental para iniciar o inspirar una investigación biomédica con el propósito de aumentar nuestros conocimientos sobre una determinada condición patológica está enfrentando en la actualidad limitaciones que podrían eventualmente restringir la publicación no sólo de estudios epidemiológicos y clínicos ya sean prospectivos o retrospectivos, sino también la presentación de casos clínicos, tan habituales en el devenir de la práctica de las ciencias médicas.

Estas restricciones se han originado en base a tres situaciones emergentes en los últimos años ${ }^{1}$ : a) la protección legal concedida a la intimidad, confidencialidad y privacidad de los datos de carácter personal; b) la progresiva informatización de datos clínicos que haría más factible la invasión de la privacidad personal por parte de terceros y c) la posibilidad de usar datos genéticos de las personas lo cual es percibido como un aumento del grado de vulnerabilidad.

Uno de los potenciales impactos de la nueva legislación en la investigación médica de nuestro país ha sido la limitación relativa al acceso a la ficha clínica ${ }^{2}$.

A comienzos de 2003 se entabló en la Revista Médica de Chile un debate académico en relación al proyecto de ley en trámite sobre derechos y deberes de las personas en salud respecto ¿A quién pertenece la ficha clínica? Por una parte se planteó que la ficha clínica es un documento privado, que le pertenece al médico o a la institución de salud a cuyo cuidado se encuentra el paciente. Se planteó también que mucho menos defendible es otorgarle el derecho a acceder a la ficha clínica a los parientes o herederos ${ }^{3}$. Por otra parte, se argumentó que si bien el médico es el responsable de elaborar la ficha clínica, este profesional podría más bien ser considerado su "custodio", siendo responsable conjuntamente con la institución de salud que trata al paciente de su correcto uso y mane$\mathrm{jo}^{4}$. Sin embargo, según esta segunda posición la única persona que puede decidir de su destino es el paciente, según el dictado del respeto a su autonomía y confidencialidad, aunque reconociendo que resulta difícil asignar un propietario específico de este documento privado ${ }^{4}$. Lo que correspondería sería condicionar su disponibilidad según una definición de las diversas funciones que cumple este documento. El papel del médico como "custodio" de la ficha clínica también fue cuestionado porque este papel puede ser desempeñado por la persona encargada del archivo institucional ${ }^{3}$.
Esta controversia fue resuelta legislativamente con la promulgación de la Ley 20.584, "Sobre derechos y deberes de los pacientes en su atención en salud"s que en su artículo 13 dispone que las personas que no estén directamente relacionados con la atención de salud del paciente no tendrán acceso a la respectiva ficha clínica, considerando las siguientes excepciones: a) titular de la ficha, su representante legal o sus herederos, estos últimos sólo son excepción tras el fallecimiento del paciente; b) un tercero autorizado ante notario por el titular; c) los tribunales de justicia (sólo si la información tiene relación con la causa que estuvieren conociendo); d) los fiscales del Ministerio Público y abogados, previa autorización del juez competente (este acceso está limitado a asuntos vinculados con la causa en investigación o defensa).

Llama la atención que entre las excepciones, del artículo 13 no se haya incluido el acceso a la información contenida en la ficha clínica para investigación biomédica, clínica o epidemiológica. Por lo tanto, el paciente debería otorgar una autorización notarial. Esta situación, podría generar un obstáculo difícil de superar para efectuar investigación médica, que resulta imprescindible para poder conocer la situación clínica y epidemiológica de diversas enfermedades y condiciones patológicas a nivel nacional ${ }^{6}$.

Esta disposición legal también podría generar una eventual limitación en la publicación de casos clínicos y de estudios retrospectivos, basados en revisión de fichas clínicas de una determinada condición que son necesarios los primeros para el conocimiento teórico y la práctica clínica y los segundos para efectuar los estudios preliminares para plantear un estudio prospectivo de mayor alcance que permita avanzar en el conocimiento de una determinada condición patológica.

En este contexto legal, los editores y los revisores de revistas nacionales estarían facultados para exigir la autorización del paciente y del Comité de Ética institucional como uno de los requisitos para aceptar la publicación de un caso clínico, condición que se agregaría a las medidas imprescindibles para proteger el anonimato del paciente en dicha publicación ${ }^{7}$.

El Ministerio de Salud (MINSAL) se pronunció sobre el procedimiento de la firma del consentimiento informado en una circular emitida el 18 de abril de 2013 titulada "Emisión de consentimiento de personas participantes de una investigación científica" en la cual la interpretación del departamento jurídico del MINSAL indica que para estos fines, las disposiciones de la ley 20.120 "Sobre la investigación científica en 
el ser humano, su genoma y prohíbe la clonación humana" 8 por ser una ley específica sobre investigación científica tienen preeminencia sobre las disposiciones de la ley 20.584 y por ello no se requiere que el consentimiento informado sea realizado ante notario. Siguiendo esta argumentación del MINSAL, para acceder al contenido de una ficha clínica con fines de investigación médica, bastaría con obtener el consentimiento informado del paciente, como sujeto de investigación ${ }^{7}$.

En relación a la percepción de cómo los Comités de Ética de Investigación enfocan el acceso a la ficha clínica para fines de investigación biomédica, un estudio realizado en Canadá ${ }^{9}$ evaluó la variabilidad de los requerimientos de los Comités de Ética de Investigación afiliados a facultades de medicina, para revisiones retrospectivas de fichas clínicas y de quienes podían tener acceso al contenido de estos registros clínicos para colectar datos. Se entrevistó a 30 comités utilizando preguntas estructuradas sobre un estudio de caso con respuestas abiertas. Se detectó una gran variabilidad en estos requerimientos. De los 30 comités $14(47 \%)$ requirieron consentimiento individual del paciente para llevar a cabo el estudio propuesto; tres $(10 \%)$ señalaron que su respuesta dependería de cómo se manejaran las potenciales variables de identificación, en tanto que 11 comités $(38 \%)$ no requirieron el procedimiento de consentimiento y los dos restantes (7\%) optaron por una notificación fuera del proceso de consentimiento. La mayoría de los comités planteó que se requeriría consentimiento, si se obtenía información identificable de los registros médicos. Se detectó preocupación de los comités sobre la posibilidad que individuos externos tuviesen acceso a las fichas clínicas. Esta preocupación estuvo asociada al requerimiento de consentimiento, lo que se reflejó en que 18 comités $(60 \%)$ exigieran una revisión completa en este caso. Finalmente 16 comités $(53 \%)$ permitieron que un asistente de investigación obtuviese información desde los registros de salud. Los autores concluyen que los Comités de Ética de Investigación entrevistados necesitan capacitación en mejores prácticas para proteger la privacidad y confidencialidad en la investigación en ciencias de la salud. Sugieren realizar foros con quienes dirigen estos comités para compartir confidencialmente preocupaciones y decisiones sobre situaciones específicas para reducir las variaciones inter-comités en la toma de decisiones ${ }^{9}$.

En relación a lo que se ha planteado en nuestro medio sobre el acceso a la ficha clínica con fines de investigación, se puede considerar la reciente proposición del Comité de Ética de Investigación en Seres Humanos de la Facultad de Medicina, Universidad de Chile 6 . Para autorizar el uso de fichas clínicas en investigación, este comité propone usar procedimientos diferentes según se trate de estudios prospectivos o retrospectivos. En los estudios prospectivos se requiere que el investigador responsable solicite consentimiento por escrito al potencial voluntario y al director de la institución o su representante. En los estudios retrospectivos se debe distinguir dos situaciones: a) si la información va a ser utilizada en forma personalizada se debe seguir el proceso habitual para obtener el consentimiento informado; b) si la información será obtenida de datos previos almacenados en forma anónima (base de datos anónima con datos identificadores encriptados) el Comité de Ética de Investigación podrá eximir de la obtención de un consentimiento informado, para lo cual deberá tomar en consideración los siguientes criterios: i. Confidencialidad de la declaración; ii. Garantizar el anonimato del paciente encriptándose los datos identificadores; iii. Compromiso y responsabilidad del investigador; iv. Valor y validez científica de la investigación; v. Justificación social y científica de la investigación propuesta ${ }^{6}$.

En este mismo contexto el Comité de Ética de Investigación del Instituto de Enfermedades Raras, del Instituto Carlos III, Madrid, España, ha propuesto directrices éticas y recomendaciones sobre la creación de registros (base de datos anonimizadas) con fines de investigación biomédica ${ }^{1}$. La creación de estos registros debería permitir realizar una investigación biomédica válida y útil respetando los principios éticos y la legalidad vigente. Las complejidades tanto de la creación de registros, como del uso de registros pre-existentes con fines de investigación biomédica, implican que estas acciones deberían ser evaluadas por un Comité de Ética de Investigación, esta evaluación es especialmente importante cuando la investigación requiera el manejo de datos de carácter personal. Si bien estas directrices ${ }^{1}$ están enmarcadas en el contexto de la legislación de España, la cual puede diferir de la legislación nacional, sus 24 recomendaciones pueden servir de marco de referencia general sobre las precauciones a considerar al crear o usar una base de datos con fines de investigación.

Entre estas recomendaciones sobre el uso de registros clínicos en investigación biomédica, merece una mención especial las recomendaciones sobre el uso de la ficha clínica y sobre el uso de registros históricos y de personas fallecidas ${ }^{1}$.

Respecto al uso de la ficha clínica se considera 
una buena práctica informar al paciente que recibe atención médica que sus datos clínicos podrían ser utilizados con fines de investigación médica y solicitarle su consentimiento para tal fin. Debería quedar constancia de dicho consentimiento en la ficha clínica. Se debe tener presente que esta práctica no exime a los investigadores de someter su investigación a la aprobación del respectivo Comité de Ética.

Sólo en circunstancias excepcionales se puede prescindir del consentimiento individual para la creación y/o uso de registros con fines de investigación que contienen información de carácter personal. En este sentido hay que hacer notar que algunas entidades (v. gr.: MINSAL, Superintendencia de Salud, FONASA: Fondo Nacional de Salud. SOME: Servicio de Orientación Médica y Estadística) que cuentan con autorización legal para acceder a la ficha clínica en razón de cumplir su función ${ }^{10}$, pueden jugar un papel importante en la creación de bases de datos clínicos de manera prospectiva, constituyendo una buena fuente autorizada de bases de datos.

En el caso que se considere la posibilidad de prescindir del consentimiento individual para acceder a datos de carácter personal, los investigadores responsables deberán esgrimir razones fundamentadas ante el Comité de Ética. Tal es el caso de los registros epidemiológicos para determinar la incidencia real de una determinada enfermedad, los cuales deben ser exhaustivos, es decir, deben incluir a la mayoría o a la totalidad de los afectados, cuestionándose la validez científica de estudios epidemiológicos cuando no se logra obtener el consentimiento informado en una proporción importante de la población afectada ${ }^{11}$. En relación al uso de registros históricos si los datos de carácter personal que contiene el registro son prescindibles, una alternativa válida es anonimizarlos. Si los datos personales son imprescindibles, habría que evaluar la posibilidad de obtener retrospectivamente el consentimiento individual, si ello no es posible, hay que considerar la posibilidad de justificar una excepción ante el Comité de Ética ${ }^{1}$.

En cuanto al uso de registros que contienen datos de carácter personal de personas fallecidas, éste puede efectuarse si hay constancia del consentimiento previo de la persona fallecida o si se obtiene el consentimiento de sus herederos. Si no se considera posible solicitarlo a los herederos el investigador puede solicitar una dispensa al respectivo Comité de Ética, el cual deberá decidir ponderando el riesgo de uso indebido de los datos y las medidas de seguridad que se establezcan ${ }^{1}$.
En todas las situaciones analizadas parece crucial el papel desempeñado por los Comités de Ética de Investigación, el cual ha sido ratificado por la nueva legislación nacional enfatizándose una vez más que su responsabilidad principal es proteger a los sujetos de investigación ${ }^{12}$.

Otra función importante de los Comités de Ética de Investigación es brindar apoyo a los investigadores en los aspectos bioéticos de sus proyectos, de manera que los investigadores biomédicos que son potenciales autores de publicaciones, no deberían considerar a estos comités como una valla o barrera que deben sortear para realizar una investigación publicable, sino que deberían considerarlos una ayuda valiosa en este delicado proceso que necesariamente debe tener una regulación ética, para prevenir los eventuales riesgos que conlleva la investigación en seres humanos que altruistamente se prestan informada y voluntariamente a actuar como sujetos de investigación.

Se debe tener presente la formación de los investigadores, ya que se ha comunicado que ningún Comité de Ética puede sustituir las cualidades éticas del investigador, por lo que se deduce que "sin investigadores éticos no hay investigación ética"13.

Se ha señalado ${ }^{14}$ que el cumplimiento de los requisitos éticos en las publicaciones biomédicas es verificado por tres filtros. El primero de ellos lo constituyen los propios autores y las autoridades que dan su aprobación inicial, quienes a su vez tienen que solicitar la aprobación del $2^{\circ}$ filtro constituido por el Comité de Ética de Investigación. El $3^{\text {er }}$ filtro lo conforma el Comité Editorial de las revistas biomédicas que reciben los manuscritos producidos por la investigación ${ }^{14}$.

Por lo tanto, los autores, revisores y editores que participan en el proceso de publicación de manuscritos en una revista biomédica deben observar y respetar los principios bioéticos que resguardan el bienestar y los derechos de los voluntarios. Este debido respeto a los principios bioéticos está declarado formalmente en las normas de publicación para los autores de las revistas biomédicas y generalmente forman parte de los requisitos que deben cumplir los manuscritos enviados a publicación, como es el caso de la Revista Chilena de Enfermedades Respiratorias ${ }^{15}$. En nuestro medio la recomendación general de la Asociación Chilena de Editores de Revistas Biomédicas (AChERB) es que en las publicaciones de estudios en seres humanos, se debe identificar a la institución o al Comité de Ética que aprobó el protocolo que originó la respectiva publicación. 


\section{Agradecimientos}

A la profesora Gina Raineri B., secretaria del Comité de Ética de Investigación en Seres Humanos, Facultad de Medicina, Universidad de Chile, por su revisión crítica de los aspectos legales de este artículo.

\section{Bibliografía}

1.- DE ABAJO IGLESIAS F J, FEITO-GRANDE L, JÚDEZ GUTIÉRREZ J, MARTÍN ARRIBAS M C, TERRACINI B, PÀMPOLS ROS T, et al. Directrices éticas sobre la creación y uso de registros con fines de investigación biomédica. Rev Esp Salud Pública 2008; 82: 21-42.

2.- O'RYAN M. Investigación biomédica en Chile a la luz de la nueva legislación. Rev Chil Infectol 2013; 1: 7-9.

3.- VACAREZZA R, NÚÑEZ E. ¿A quién pertenece la ficha clínica? Rev Med Chile 2003, 131: 111-4.

4.- ROSSELOT E. ¿A quién pertenece la ficha clínica? Rev Med Chile 2003; 131: 815-6 (Carta al editor).

5.- GOBIERNO DE CHILE. Ley 20.584. Sobre derechos y deberes de los pacientes en su atención en salud. 13 de abril de 2012.

6.- OYARZÚN M, PINTO M E, RAINERI G, AMIGO H, CIFUENTES L, GONZÁLEZ M J, et al. Experiencia del Comité de Ética de Investigación en Seres Humanos de la Facultad de Medicina de la Universidad de Chile y los desafíos que impone la nueva legislación chilena en la investigación médica. Rev Med Chile 2014; 142 : 889-95.

7.- RAINERI G, OYARZÚN M. Potencial impacto de la nueva legislación en la investigación biomédica en
Chile. Rev Chil Enferm Respir 2013; 29: 5-8 (Editorial).

8.- GOBIERNO DE CHILE. Ley 20.120 "Sobre la investigación científica en el ser humano, su genoma y prohíbe la clonación humana", 7 de septiembre de 2006.

9.- WILLISON D J, EMERSON C, SZALA-MENEOK $\mathrm{K} \mathrm{V}$, GIBSON E, SCHWARTZ L, WEISBAUM $\mathrm{K} \mathrm{M}$, et al. Access to medical records for research purposes: varying perceptions across research ethics boards. J Med Ethics 2008; 34; 308-14. doi: 10.1136/ jme.2006.020032.

10.- CONTRALORÍA GENERAL DE LA REPÚBLICA. Dictamen $N^{\circ} 19652$ Sobre los sujetos autorizados a acceder a la ficha clínica de un paciente. 2 de abril de 2013.

11.- TU J, WILLISON D J, SILVER F L, FANG J, RICHARDS J A, LAUPACIS A, et al. Impracticability of informed consent in the registry of the Canadian Stroke Network. N Engl J Med 2004; 350: 1414-21.

12.- TÉLLEZ R. El rol de los Comités de Ética de la investigación en el nuevo marco legal. En: Impacto de la nueva normativa legal sobre la investigación científica en Chile" $8^{\circ}$ Taller de Bioética. Comité Asesor de Bioética FONDECYT de CONICYT. Santiago, diciembre de 2012, p. 39-45 ISBN 978-956-7524 18-1.

13.- VACAREZZA R. Los Comités de Bioética en Chile: $\mathrm{Su}$ rol y realidad de funcionamiento En: Bravo M. Reflexiones sobre Bioética: Seminarios de la Academia Chilena de Medicina (2011-2013). Santiago, 2015. p. 163-9.

14.- REYES H. Ética de las publicaciones científicas. En: Bravo M. Reflexiones sobre Bioética: Seminarios de la Academia Chilena de Medicina (2011-2013). Santiago, 2015. p. 120-8.

15.- NORMAS DE PUBLICACIÓN PARA LOS AUTORES. Rev Chil Enferm Respir 2015; 31: 181-2.

Correspondencia a:

Dr. Manuel Oyarzún Gómez

Programa de Fisiopatología, ICBM,

Facultad de Medicina, Universidad de Chile.

Avda. Salvador 486, Providencia.

Código postal 7500922 Correo 9. Providencia

Email: moyarzun@med.uchile.cl 